\title{
Facilitated Peptide Transport via the Mucosal Epithelium: Impact on Tolerance Induction
}

\author{
Elisabeth E. Kenngott ${ }^{1,2}$, Jennifer Pfeil ${ }^{1,3}$, Ute Hoffmann', Uta Lauer ${ }^{1}$, Anja A. Kühl', \\ Anne Rigby ${ }^{3}$, Anton Pernthaner ${ }^{5}$ and Alf Hamann ${ }^{1,3 *}$
}

\begin{abstract}
'Experimental Rheumatology, Deutsches Rheuma-Forschungszentrum, Berlin, Germany, ${ }^{2}$ Rheumatology, Department of Gastroenterology, Infectiology and Rheumatology, Charité Universitätsmedizin Berlin, Berlin, Germany, ${ }^{3}$ Experimental Rheumatology, Department of Rheumatology and Clinical Immunology, Charité Universitätsmedizin Berlin, Berlin, Germany, ${ }^{4}$ Medical Department, Division of Gastroenterology, Infectiology and Rheumatology, Research Center ImmunoSciences, Charité Universitätsmedizin Berlin, Berlin, Germany, ${ }^{5}$ The Hopkirk Research Institute, AgResearch Ltd., Grasslands Research Institute, Palmerston North, New Zealand
\end{abstract}

\section{OPEN ACCESS}

Edited by: Rachel R. Caspi, National Institutes of Health (NIH),

Reviewed by: Eduardo Villablanca, Karolinska Institutet, Sweden Arya Biragyn, National Institutes of Health $(N I H)$,

*Correspondence: Alf Hamann hamann@drfz.de

Specialty section: This article was submitted to Immunological Tolerance and Regulation, a section of the journa Frontiers in Immunology

Received: 14 September 2016 Accepted: 15 February 2017 Published: 06 March 2017

Citation: Kenngott EE, Pfeil J, Hoffmann U, Lauer U, Kühl AA, Rigby A, Pernthaner A and Hamann A (2017) Facilitated Peptide Transport via the Mucosal Epithelium: Impact on Tolerance Induction. Front. Immunol. 8:216. doi: 10.3389/fimmu.2017.00216
A hallmark of autoimmunity is the breakdown of tolerance and generation of effector responses against self-antigens. Re-establishment of tolerance in autoimmune disorders was always the most desired treatment option; however, despite many efforts, clinical trials have been largely unsuccessful. This also applies to the generation of oral tolerance, which seems to be a default response type of the mucosa-associated lymphoid tissues to harmless antigens. In this study, we report improved efficacy of oral tolerance induction by coupling antigen with the newly identified mucosal carrier peptide $13 \mathrm{C}$. Antigen coupled to $13 \mathrm{C}$ is efficiently taken up in the gastrointestinal tract and could be visualized in cells of the lamina propria. Oral, rectal, or nasal treatment effectively induced the proliferation of antigen-specific $T$ cells with some increase in the frequency of regulatory $T$ cells. In a model of delayed-type hypersensitivity, especially intrarectal tolerization treatment resulted in reduced footpad swelling, demonstrating a moderate tolerogenic effect of mucosal treatment with 13C coupled antigen. Coupling of antigens to a transmucosal carrier, therefore, is a promising tool to improve the efficacy of vaccination via mucosal surfaces.

Keywords: oral tolerance, peptide vaccination, targeted delivery, mucosa, regulatory T cells, mucosal uptake

\section{INTRODUCTION}

Mucosal surfaces are constantly exposed to the external environment and are main entry sites for pathogens. The gut mucosa discriminates between pathogens on the one side and harmless food antigens and commensals on the other side, with antigens from the latter categories rather eliciting ignorance or active tolerance in the immune system than inducing effector mechanism.

Whether a foreign antigen induces immunity or tolerance is determined by intrinsic and conditional factors: the recognition of antigen or its carrier by receptors of the innate immune system, such as toll-like receptors, determines the outcome of the response. Conditions where inflammatory signals are abundant, either in the environment or on purpose by vaccine adjuvants, lead to the generation of strong effector responses. In contrast, antigens that do not stimulate the innate system, such as soluble antigens or peptides, appear to elicit induction of tolerance as a default response. Notably, oral administration of soluble antigens has been shown to induce systemic unresponsiveness and 
can be mediated by the induction of regulatory cells or by clonal deletion and/or induction of anergy in effector cells, a response designated as oral tolerance $(1,2)$.

Manipulation of induction of oral tolerance is seen as an attractive means to induce antigen-specific tolerance to prevent autoimmune diseases. However, to date, clinical trials have largely been unsuccessful (3). This might depend on two major issues: first, pro-inflammatory conditions, prevalent in patients affected with autoimmune disease might suppress successful establishment of tolerance. It appears conceivable, however, that concomitant inhibition of inflammatory pathways might overcome this issue in future approaches. Second, vaccination via the oral route is of poor efficacy due to poor uptake and rapid degradation of antigen in the gastrointestinal tract, in particular when peptides are used. In the present study, we investigated if coupling of antigenic peptides to a specific cyclic peptide, which facilitates transportation through the gut epithelium, promotes selective uptake, and thereby induction of tolerance.

We recently identified the $13 \mathrm{C}$ peptide that increases uptake and transcytosis of peptides and proteins upon mucosal administration (4). The study demonstrated that labeled $13 \mathrm{C}$ peptide as well as peptide or protein bound to it is rapidly taken up by subsets of epithelial cells in the intestinal mucosa. These cells display the morphology of goblet cells which already have been implicated in the spontaneous uptake of antigens $(5,6)$. The constructs were then either transported into lymph and subsequently into systemic circulation, or taken up by CD11c-positive cells, most likely dendritic cells (DCs), of the lamina propria and Peyer's patches. The 13C peptide, therefore, is considered a promising candidate for the delivery of antigens to the immune compartment for the induction of tolerance via the intestinal route.

In the present work, we assessed the tolerogenic potential of mucosal vaccinations using $13 \mathrm{C}$ peptide-coupled ovalbumin peptide (OVA323-339: pOVA) and tested T cell responses in the DO11.10 transfer model system (7). Antigen-induced proliferation, induction of Foxp $3^{+}$Tregs, production of IL-10, TNF, and other cytokines was analyzed in reactive $\mathrm{T}$ cells, and the impact of mucosal vaccination in a delayed-type hypersensitivity $(\mathrm{DTH})$ reaction was measured to detect dominant tolerance. We found that oral, rectal, and intranasal administration of 13C-pOVA peptide significantly increased proliferation of antigen-specific $\mathrm{T}$ cells. Increased frequencies of regulatory $\mathrm{T}$ cells as well as a shift of the ratio of IL-10 to TNF in favor of IL-10 was observed in some conditions. Especially intrarectal application of $13 \mathrm{C}$ coupled to pOVA resulted in a reduced footpad swelling in the DTH model. Thus, the use of the described targeting approach might be useful to improve oral tolerization protocols.

\section{MATERIALS AND METHODS}

\section{Mice}

Female Balb/c mice were purchased from Charles River (Sulzfeld, Germany) and used at 8-10 weeks of age. DO11.10 pOVA-TCR transgenic mice extensively backcrossed ( $>10$ generations) onto Balb/c background were housed in the DRFZ breeding facility. All mice were maintained under specific pathogen-free conditions according to national and institutional guidelines. All experiments were approved by Landesamt für Gesundheit und Soziales (LAGeSo; G0014/14).

\section{Peptide Synthesis}

OVA peptide 323-339 (pOVA: ISQAVHAAHAEINEAGR), 13CpOVA peptide (CTANSSAQC- $\beta$ Ala-ISQAVHAAHAEINEAGR), and 13Csc-pOVA-peptide (scrambled control; CSNAQATSC$\beta$ Ala-ISQAVHAAHAEINEAGR) were synthesized in house (Institute for Medical Immunology, Charité Universitätsmedizin Berlin, Germany) as described previously. 13C-FITC and 13CscFITC were synthesized by jpt peptides (Berlin, Germany). All $13 \mathrm{C}$ or $13 \mathrm{Csc}$ constructs were cyclysed using $10 \%$ DMSO (v/v) in $\mathrm{H}_{2} \mathrm{O}$ with a $\mathrm{pH} 8-8.5$.

\section{Cell Preparation}

Single cell suspensions were prepared from lymph nodes and spleen. Red blood cells were lysed in lysis buffer [0.01 M $\mathrm{KHCO}_{3} ; 0.155 \mathrm{M} \mathrm{NH}{ }_{4} \mathrm{Cl}, 0.1 \mathrm{mM}$ ethylenediaminetetraacetic acid (EDTA)] and washed with phosphate buffered saline (PBS) containing $0.2 \%$ BSA. CD $4^{+}$T cells were enriched using anti-CD4 microbeads (clone: RM4-5, Miltenyi Biotec, Bergisch Gladbach, Germany) according to manufacturer's instructions and sorted using an AutoMACS Pro (Miltenyi Biotec). Cell enrichment was verified by flow cytometric analysis; a frequency of $>94 \%$ $\mathrm{CD}^{+}$cells was considered successful. Generally, the frequency of pOVA-specific cells $\left(\mathrm{KJ} 1.26^{+}\right)$was between $70-80 \%$ within the $\mathrm{CD}^{+}$cell pool.

\section{In Vitro Proliferation Assay}

For in vitro analysis, $\mathrm{CD}^{+}$cells from DO11.10 mice were labeled with $1 \mu \mathrm{M}$ CFSE in PBS. The CD4-negative fraction was depleted from the remaining T cells using anti-CD90 microbeads (Miltenyi Biotec) and AutoMACS separation and used as antigen-presenting cells (APCs). APCs were irradiated (30 Gy for $28 \mathrm{~min}$ ) and cultured with $\mathrm{CD} 4^{+}$cells in a final concentration of $2 \times 10^{6}$ cells/ ml cRPMI [RPMI 1640 Glutamax medium (Gibco, Paisley, UK) supplemented with $10 \%$ fetal calf serum, penicillin $(100 \mathrm{U} / \mathrm{ml})$, streptomycin $(100 \mu \mathrm{g} / \mathrm{ml}), 2$-mercaptoethanol $(1 \mathrm{mM})$, sodium pyruvate $(1 \mathrm{mM})$, and HEPES ( $\mathrm{N}$-2-hydroxyethylpiperazine$N^{\prime}$-2-ethanesulfonic acid; $25 \mathrm{mM}$ )] with a ratio of 4:1 in 96-well round-bottom plates. pOVA constructs were added in various concentrations as indicated. Cells were incubated for 4 days at $37^{\circ} \mathrm{C}$ in a humidified $5 \% \mathrm{CO}_{2}$ atmosphere. For analysis of proliferation, cells were stained and analyzed by flow cytometry by gating on pOVA-TCR ${ }^{+} \mathrm{CD}^{+}$cells and calculating the geometrical mean of the fluorescence intensity (GMFI) of the CFSE signal. Fold CFSE dilution was determined [fold CFSE dilution: GMFI (PBS control)/GMFI (sample)].

\section{In Vivo Proliferation Assay}

Purified total CD4 ${ }^{+}$CFSE-labeled cells isolated from peripheral lymph nodes and spleens of DO11.10 mice were adoptively transferred i.v. into Balb/c mice $\left(5 \times 10^{6}\right.$ cells/mouse). On the following day, mice were treated with peptide-pOVA constructs (pOVA, 13C-pOVA or13Csc-pOVA) or PBS, either i.v., orally, 
rectally or nasally as indicated. For oral treatment, mice were fed intragastral with $0.5 \mathrm{mg}$ peptide or equimolar amounts of peptide conjugates in $200 \mu \mathrm{l}$ PBS using a feeding needle (FST, Heidelberg, Germany). For rectal treatment, mice were injected rectally with $0.5 \mathrm{mg}$ pOVA or equimolar amounts of peptide-conjugates (unless otherwise indicated) in $200 \mu \mathrm{l}$ PBS using an umbilical catheter with a $0.5 \mathrm{~mm} \times 0.8 \mathrm{~mm}$ diameter. For nasal treatment, mice were anesthetized with $100 \mu \mathrm{l}$ Dexdomitor $(0.4 \mathrm{mg} / \mathrm{ml}$, Pfizer, New York, NY, USA) and short isoflourane (Abbott, Chicago, IL, USA) inhalation. After intranasal application of $0.05 \mathrm{mg}$ pOVA or equimolar amounts of peptide conjugates in $20 \mu \mathrm{lBS}$, mice were injected with $100 \mu \mathrm{l}$ Antisedan (2 mg/ml, Pfizer).

\section{DTH Reaction}

Balb/c mice received a transfer of $\mathrm{CD}^{+}$cells from DO11.10 donor mice and were tolerized intrarectally as described before in the in vivo proliferation assay. On day 8 after cell transfer, the mice received a subcutaneous (tailbase) injection of pOVA (125 $\mu \mathrm{g})$ in complete Freund's adjuvans [complete Freund's adjuvant (CFA); Sigma-Aldrich, St. Louis, MO, USA]. To induce a strong DTH reaction on day 22 , the mice were injected into the right footpad with $250 \mathrm{ng}$ of pOVA in $5 \mu \mathrm{l}$ of incomplete Freund's adjuvans [incomplete Freund's adjuvant (IFA), Sigma-Aldrich]. PBS/IFA emulsion was injected into the left footpad as a control. The footpad swelling was determined $24 \mathrm{~h}$ after challenge using an Oditest micrometer gauge (Kroeplin Längenmesstechnik, Schlüchtern, Germany).

\section{Antibodies and Flow Cytometry}

The following antibodies and reagents were obtained from eBioscience (San Diego, CA, USA): eFlour 450-conjugated antiCD4 (RM4-5), PE conjugated anti-IL-10 (JES5-16E3) PE-Cy7 conjugated anti-IFN $\gamma$ (XMG1.2), PerCP-eFlour 710 conjugated anti-TNFo (MP6-XT22), eFlour 450-conjugated anti-Foxp3 (FJK-16s), and appropriate isotype controls. V500-conjugated anti-CD4 (RM4-5) antibody was purchased from BD Bioscience (Heidelberg, Germany). Cy5-conjugated anti-pOVA-TCR antibody (KJ1.26) and anti-Fcy-receptor antibody (2.4G2) were produced in house (Deutsches Rheuma-Forschungszentrum Berlin). Total rat IgG was purchased from Dianova (Hamburg, Germany).

Cell surface staining of lymphocytes was performed in the presence of anti-Fc $\gamma$-receptor antibody $(20 \mu \mathrm{g} / \mathrm{ml})$. Intracellular Forkhead-box-protein 3 (Foxp3) staining was performed using the anti-mouse Foxp3 staining set (eBioscience, San Diego, CA, USA) according to manufacturer's instructions.

For cytokine staining, cells were stimulated with phorbol12-myristate 13 -acetate (PMA $10 \mathrm{ng} / \mathrm{ml}$ ) and ionomycin (500 ng/ $\mathrm{ml}$ ) (Sigma-Aldrich) for $2 \mathrm{~h}$ at $37^{\circ} \mathrm{C}$ in a humidified $5 \% \mathrm{CO}_{2}$ atmosphere. Brefeldin A $(10 \mu \mathrm{g} / \mathrm{ml})$ (Sigma-Aldrich) was added, and cells were incubated for another $2 \mathrm{~h}$. After surface staining, cells were fixed by incubation with $2 \%$ paraformaldehyde (PFA). Intracellular staining was performed in PBS containing 0.5\% saponin (Sigma-Aldrich) after 5 min pre-incubation with blocking rat IgG. Flow cytometry was performed using a FACS Canto II (BD Bioscience) and analyzed with Flow Jo software (TreeStar, Ashland, OR, USA).

\section{Microscopical Analysis}

For visualization of peptide uptake, BALB/c mice were anesthetized by intraperitoneal (i.p.) injection of Ketamin/Xylazin solution (200 and $10 \mathrm{mg} / \mathrm{kg}$, respectively; Ketavet ${ }^{\circledR}$, Zoetis, Berlin, Germany; Rompun ${ }^{\circledR}$, Bayer, Leverkusen, Germany) and fluorescein isothiocyanate (FITC) coupled peptide construct were injected into a ligated loop of the large intestine $(0.02 \mu \mathrm{g} / \mu \mathrm{l})$. A $1.5-\mathrm{cm}$ loop was injected with approximately $100 \mu \mathrm{l}$. After 10 min incubation, the tissue was removed and fixed in $2 \%$ PFA solution for approximately $16 \mathrm{~h}$. For dehydration, the tissue was incubated in increasing solutions of sucrose (10,20, and 30\%), for 2 days at $4^{\circ} \mathrm{C}$. After tissue embedding and freezing, $7 \mu \mathrm{m}$ cryostat sections were made using the HM 560 Cryotom (Thermo Fischer Scientific, Waltham, MA, USA). Slides were frozen at $-20^{\circ} \mathrm{C}$ until further use.

For immunohistochemistry, the slides were rehydrated in PBS. Washing steps were performed using PBS containing 1\% BSA and $0.1 \%$ Tween 20 . The blocking buffer additionally contained $10 \%$ rat serum. After incubation with blocking buffer, the sections were stained with anti-CD11c mAb (clone N418; produced in-house) conjugated to AlexaFlour ${ }^{\circledR} 647$ and washed twice. Immediately before analysis, the slides were mounted with DAKO mounting medium containing DAPI (4,6-diamino-2-phenylindole; SigmaAldrich). The slides were analyzed using a LSM 710 confocal microscope (Carl Zeiss, Jena, Germany), and pictures were taken with the software Zen 2011 (Carl Zeiss MicroImaging GmbH, Göttingen, Germany).

For histopathological analysis in the DTH mouse model, paws were fixed in formalin, bone material was decalcified employing $0.5 \mathrm{M}$ EDTA pH8 and whole paws were embedded in paraffin. Paraffin sections were cut (1-2 $\mu \mathrm{m})$, dewaxed, and stained histochemically with hematoxylin and eosin (H\&E) for overview. $H \& E$ stained sections were evaluated for epidermal changes like immune cell infiltration, spongiosis, and scurf as well as for inflammation of the dermis.

\section{Statistics}

Data were analyzed using Prism 5 (GraphPad, La Jolla, CA, USA). Statistical tests include: Mann-Whitney test, followed by Holm-Bonferroni correction for multiple comparisons, multiple comparison analysis using Kolmogorov-Smirnov and D’Agostino and Pearson omnibus test, non-parametric Kruskal-Wallis test followed by Dunn's post test and One-way ANOVA followed by Tukey's or Bonferroni's post test. Statistical analysis of cell proliferation was calculated using the fold-change values. Differences were considered as statistically significant with $p \leq 0.05\left(^{*}\right)$, very significant with $p \leq 0.01\left(^{* *}\right)$ and extremely significant with $\left.p \leq 0.001{ }^{* * *}\right)$ or marked as non-significant (n.s.).

\section{RESULTS}

\section{Coupling of $13 \mathrm{C}$ to pOVA Peptide Does Not Alter Its Recognition In Vitro and In Vivo}

An in vitro proliferation assay was employed to verify that modification of pOVA by conjugation to the cyclic 13C peptide does 
not impair presentation by APCs and recognition by antigenspecific T cells. pOVA or equimolar amounts of 13C-pOVA were tested in a 4-day culture of APCs (CD4-CD90-) and carboxyfluorescein succinimidyl ester (CFSE)-labeled pOVA-specific $\mathrm{CD}^{+}$ $\mathrm{T}$ cells from DO11.10 mice. The gating strategy is available in Supplementary Material (Figures S1A,B in Supplementary Material). We found that $\mathrm{T}$ cell proliferation and dose response curves were similar for pOVA and 13C-pOVA (Figure S2A in Supplementary Material). Coupling of 13C to pOVA, therefore, did not change the capacity of the model antigen for $\mathrm{T}$ cell activation. This was also verified in in vivo experiments. One day prior to the peptide treatment, $\mathrm{CD} 4^{+}$cells from DO11.10 donor mice were adoptively transferred into Balb/c mice. Intravenous (i.v.) injection of $5 \mu \mathrm{g}(2.8 \mathrm{nmol})$ pOVA, equimolar amounts of 13C-pOVA, or of a control peptide with a scrambled amino acid sequence coupled with pOVA (13Csc-pOVA) led to similar levels of $\mathrm{T}$ cell proliferation in the spleen, further confirming that pOVA is fully functional when conjugated to either $13 \mathrm{C}$ or $13 \mathrm{Csc}$ (Figure S2B in Supplementary Material).

\section{Mucosal Administration of 13C-pOVA Leads to Increased Proliferation of Antigen-Specific Cells}

To assess whether coupling of $13 \mathrm{C}$ to pOVA improves $\mathrm{T}$ cell responses upon administration via different mucosal routes, Balb/c mice adoptively transferred with CFSE-labeled pOVA$\mathrm{TCR}^{+} \mathrm{CD} 4^{+} \mathrm{T}$ cells received pOVA or equimolar amounts of peptide conjugates by mucosal application. We found that mucosal application of 13C-pOVA resulted in significantly enhanced $\mathrm{T}$ cell proliferation when compared to pOVA. To assess whether the facilitated transport is restricted to distinct tissues, we applied the conjugates intragastrically ("oral"), rectally, and nasally. The proliferation of antigen-specific $\mathrm{T}$ cells was increased after treatment with 13C-pOVA compared to pOVA alone (Figure 1), albeit the effect differed in strength and occurrence among the immune tissues and delivery routes tested. Intranasal application of 13C-pOVA induced the highest responses overall, with significantly higher proliferation in all three analyzed organs, mediastinal (MedLN) and mesenteric lymph nodes (MLN), and spleen, compared to the PBS-treated group (Figure 1C). Similarly, oral application of 13C-pOVA significantly increased proliferation in the MLN as well as in subcutaneous LN (SLN; pooled axillary, brachial, and inguinal LN) compared to PBS treatment (Figure 1B). Rectal application of 13C-pOVA increased the proliferation especially in the MLN (Figure 1D). We conclude that the use of the 13C peptide as transporter module results in an increase of in vivo $\mathrm{T}$ cell responses to peptide antigen administered via the oral, rectal, and nasal route.

The antigen-specific $\mathrm{T}$ cell response was evaluated in greater detail after intrarectal treatment with pOVA and conjugates of $13 \mathrm{C}$ and 13Csc with pOVA. A dose-dependent increase in proliferation was seen for 13C-pOVA which was significantly higher than for pOVA or 13Csc-pOVA, the scrambled control peptide (Figure 2).

To visualize the uptake efficiencies in the large intestine, 13C and $13 \mathrm{Csc}$ peptides were coupled with FITC and introduced into a ligated loop of the colon (colon ascendens/colon transversum). 13C-FITC was efficiently transported into cells of the lamina propria of the large intestine, already $10 \mathrm{~min}$ after injection (Figures 3A,D-F). In contrast, the control conjugate 13Csc-FITC could not be observed in the tissue (Figures 3C,D). Immunofluorescence analyses revealed that epithelial cells had taken up 13C-FITC, but also some of the subjacent CD11cpositive cells in the lamina propria as well as in isolated lymphoid follicles.

\section{Mucosal Application of 13C-pOVA Leads to a Moderate Induction of Regulatory T Cells and Significantly Decreases the Frequency of Effector T Cells}

To analyze whether transporter-mediated mucosal uptake of peptide induces a tolerogenic response as described for some oral immunization protocols [reviewed in Ref. (8)], we analyzed the expression of Foxp3, the master transcription factor of Tregs, as well as the cytokine expression of antigen-specific T cells that became activated upon mucosal application of the peptides.

Intrarectal administration of 13C-pOVA significantly increased frequencies of Foxp $3^{+}$cells 3 days after application which in the majority are also $\mathrm{CD} 25^{+}$(Figures 4A,B; Figure S4 in Supplementary Material). The increase was not due to enhanced deletion of non-Tregs since the frequency of antigen-specific cells remained unchanged compared to control animals (Figure S3 in Supplementary Material). Six days after intrarectal peptide application, frequencies of Foxp $3^{+}$Tregs were similar in pOVA and 13C-pOVA-treated animals (Figure S5F in Supplementary Material). Contrarily, after oral or nasal treatment, the increase in Foxp3 positive cells in the draining lymph nodes (MLN and MedLN, respectively) was small at day 3 but increased until day 6 (Figures 5A,F; Figures S5A-D in Supplementary Material).

Animals treated with 13C-pOVA rectally exhibited higher frequencies of IL-10 producing $\mathrm{T}$ cells compared to treatment with pOVA (Figures 5D,I). As tolerance can also rely on the induction of anergy in antigen-specific cells, we measured the percentage of $\mathrm{T}$ cells producing the inflammatory cytokines TNF and IFN $\gamma$. Frequencies of $\mathrm{TNF}^{+} \mathrm{T}$ cells appeared to be reduced upon mucosal application of 13C-pOVA (Figures 5B,G), but these differences were not statistically significant $(p=0.23)$ due to high experimental variability. IFN $\gamma$-producing cells were generally very low in numbers and unchanged upon peptide treatment (Figures $\mathbf{5} \mathbf{C}, \mathbf{H}$ ). The calculation of the ratio of IL-10 to TNF-producing cells in individual mice revealed a highly significant bias toward an anti-inflammatory cytokine milieu in 13C-pOVA-treated animals (Figure 5E). We could not observe increased IL-10 frequency after oral treatment in MLN (Figure S6 in Supplementary Material). The IL-10 production in MedLN after nasal treatment could not be measured due to technical reasons.

In none of the mice we detected significant numbers of interferon gamma (IFN $\gamma$ )-producing $\mathrm{T}$ cells under the conditions used here. We conclude that the facilitated mucosal transport of peptide antigen increases the frequency of Foxp $3^{+}$Tregs. After 
A

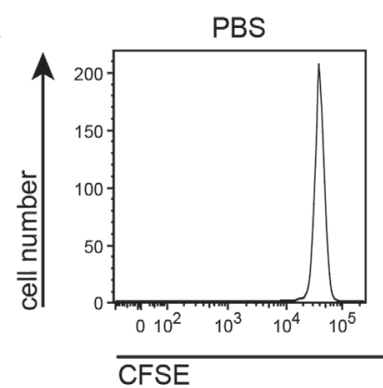

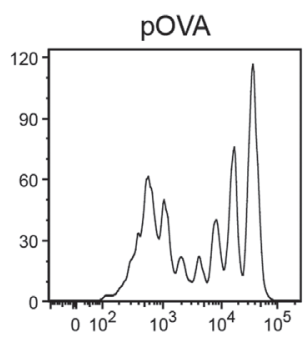

pOVA

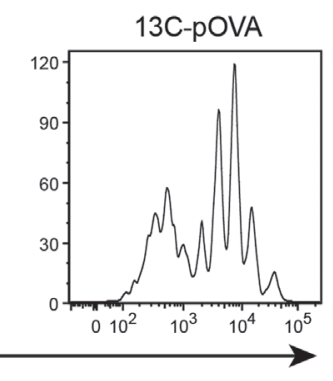

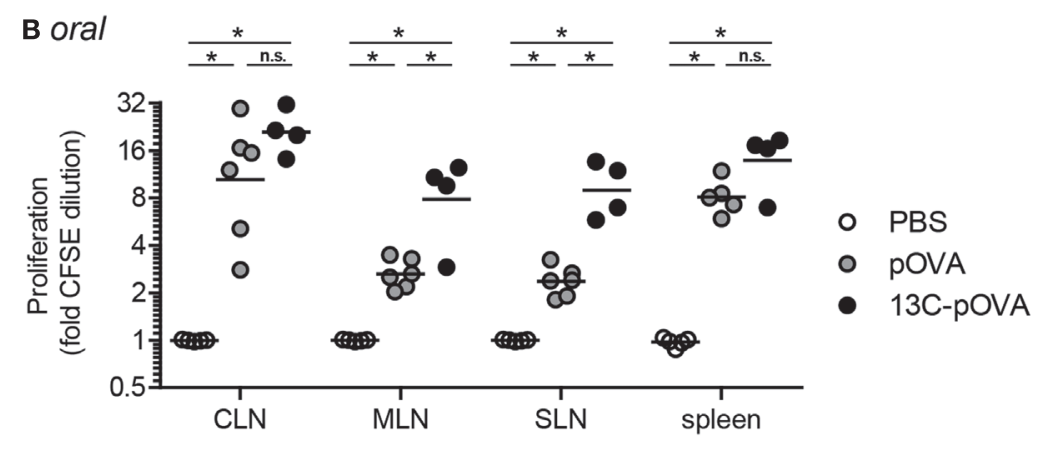

C nasal

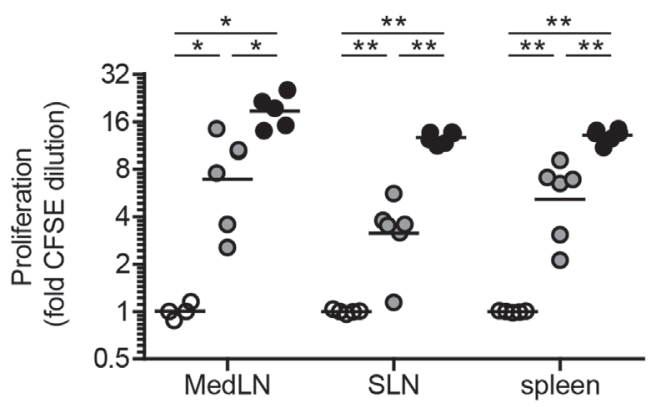

D rectal

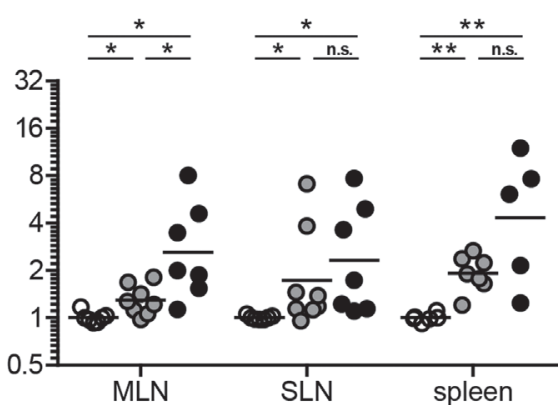

FIGURE 1 | Coupling 13C to pOVA significantly enhances proliferation of antigen-specific cells upon mucosal application. Balb/c mice were injected with $5 \times 10^{6} \mathrm{CFSE}$-labeled CD4+ cells from DO11.10 donor mice. Twenty-four hours later, mice were treated mucosally with pOVA, 13C-pOVA, or phosphatebuffered saline (PBS) as control. After 3 days, animals were sacrificed and the proliferation of pOVA-TCR ${ }^{+} C D 4^{+} T$ cells in different organs was analyzed by flow cytometry. (A) Representative histograms showing the CFSE dilution in the spleen after oral treatment with PBS, $0.5 \mathrm{mg}(0.28 \mu \mathrm{mol})$ pOVA, or equimolar amounts of 13C-pOVA. (B) T cell proliferation of mice, treated orally with $0.5 \mathrm{mg}$ pOVA or equimolar amounts of 13C-pOVA. (C) Mice received $0.05 \mathrm{mg}$ pOVA peptide or equimolar amounts of 13C-pOVA intranasally (nasal). (D) Mice were injected intrarectally with $0.5 \mathrm{mg}$ pOVA peptide or equimolar amounts of 13C-pOVA. Pooled data from two to three individual experiments with the Geometric Mean are shown; symbols represent values from individual mice $(n=4-8)$. Statistical testing was performed for each individual organ using the non-parametric Mann-Whitney test and Holm-Bonferroni correction for multiple comparisons. The complete statistical results are shown in Table S1 in Supplementary Material. CLN, cervical lymph nodes; MLN, mesenteric lymph nodes; SLN, subcutaneous lymph nodes; MedLN, mediastinal lymph nodes.

intrarectal treatment, the cytokine response was shifted toward the anti-inflammatory cytokine IL-10.

\section{Intrarectal Tolerization with 13C-pOVA Reduces Inflammation in a Model of DTH}

To further investigate the tolerogenic potency of the 13C-pOVA peptide treatment, the peptide derivatives were tested in a DTH mouse model. Seven days after intrarectal peptide treatment, mice adoptively transferred with DO11.10 cells were injected subcutaneously with pOVA in CFA. Two weeks later, the footpad of the right hind leg was challenged by subcutaneous injection of pOVA in IFA and footpad swelling measured $24 \mathrm{~h}$ later. Animals pretreated intrarectally with 13C-pOVA peptide showed a significantly reduced footpad swelling, while DTH was not affected in mice pretreated with pOVA or 13Csc-pOVA (Figure 6). In addition, the frequency of pOVA-TCR ${ }^{+} \mathrm{CD} 4^{+}$cells in the draining popliteal lymph nodes was analyzed. The foot injected with pOVA/IFA emulsion showed significantly increased infiltration of antigen-specific $\mathrm{CD}^{+} \mathrm{T}$ cells compared to $\mathrm{PBS}$, yet frequencies were not affected by the pretreatments (Figure S7 in Supplementary Material). Frequencies of IFN $\gamma$ and IL-17producing cells were low among the cells and not changed by the treatment (Figure S8 in Supplementary Material). Additional data from oral and nasal vaccination experiments supported a 
trend of reduced footpad swelling, increased frequencies of Tregs, and improved histopathology (Figure S9 in Supplementary Material). Taken together, these experiments demonstrated that

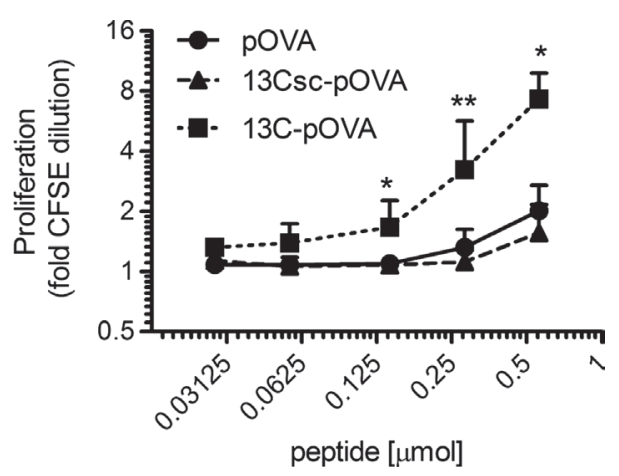

FIGURE 2 | Intrarectal treatment with 13C-pOVA peptide enhances proliferation of antigen-specific T cells. Balb/c mice were injected with $5 \times 10^{6}$ CFSE-labeled CD4+ T cells from D011.10 donor mice. Twenty-four hours later, mice were treated intrarectally with indicated amounts of pOVA peptide or equimolar amounts of 13Csc-pOVA or 13C-pOVA. After 3 days, animals were sacrificed and pOVA-TCR ${ }^{+} \mathrm{CD} 4^{+} \mathrm{T}$ cells in the mesenteric lymph nodes were analyzed by FACS. Pooled data from one to three individual experiments are shown $(n=4-8$; Geo. mean \pm SD). Statistical testing was performed for each time point using the non-parametric Kruskal-Wallis test. mucosal pretreatment with pOVA coupled to the transporter $13 \mathrm{C}$ is inducing partial tolerance in this animal model.

\section{DISCUSSION}

The gut environment features a number of different tolerogenic mechanisms, including high numbers of Tregs in the lamina propria that contribute to the tendency of mucosal immune reactions that result, in the absence of danger signals, in tolerance (9-11). For a long time, the induction of oral tolerance was seen as an attractive goal for therapeutic application, yet clinical trials have not been successful so far for several reasons (3). Intact proteins have mostly been used for vaccination, however, are difficult to standardize. In contrast, peptides are well-defined and more convenient regarding quality control. For both peptides and proteins, the gastrointestinal immunization route with its limited uptake efficiency and rapid degradation is a major obstacle.

The present study targets the issue of poor uptake of gastrointestinal vaccines by application of a novel transmucosal transporter peptide that was previously found to carry cargo through the mucosal barrier. The $13 \mathrm{C}$ peptide, a nine amino acid long cyclic peptide facilitated mucosal uptake of coupled molecules by, as of yet, unknown mechanisms (4). We here studied the impact of this strategy on vaccination efficacy and tolerance induction in the DO11.10/OVA323-339 peptide (pOVA) model. Oral, rectal, and
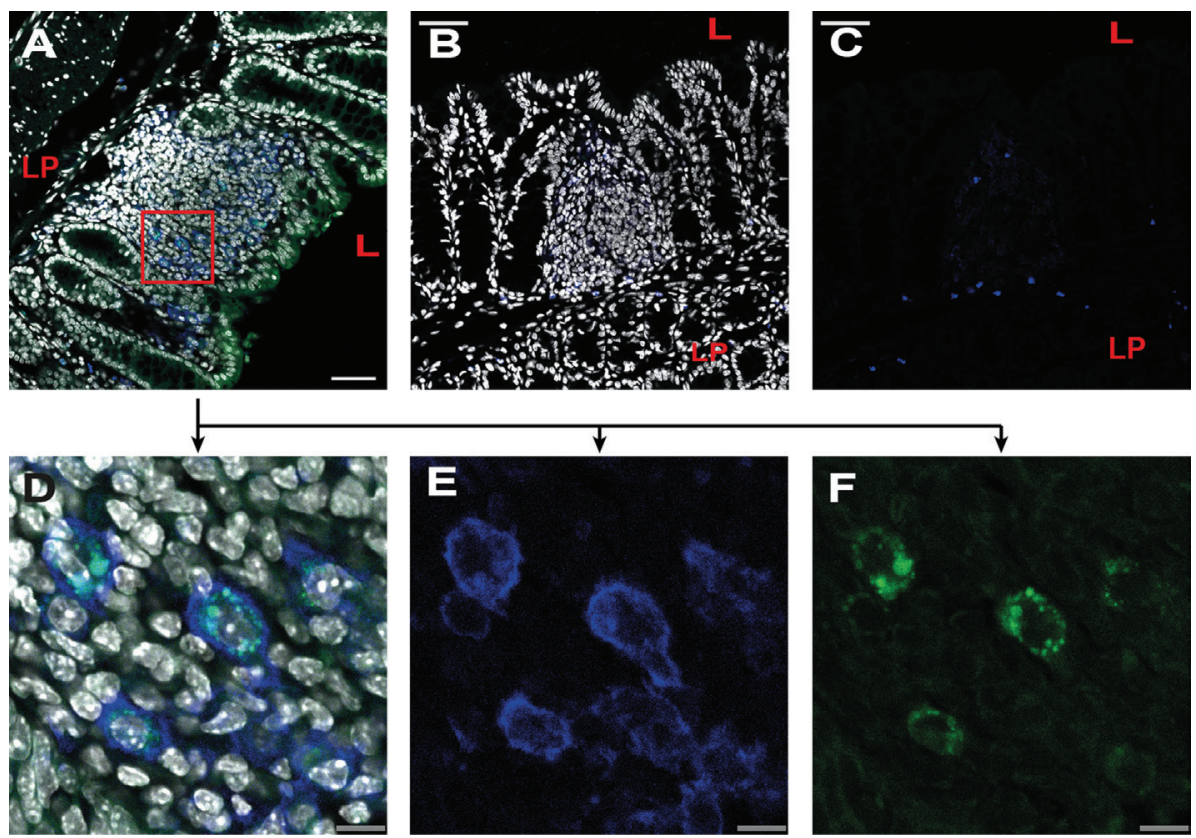

FIGURE 3 | 13C peptide is taken up by epithelial cells and CD11c-positive cells in the large intestine. Balb/c mice were anesthetized and injected with fluorescein isothiocyanate (FITC)-labeled peptides into a ligated loop of the large intestine. After 10 min, mice were sacrificed and the tissue fixed and frozen. Cryosectioned slides were stained with CD11C-Alexa647 (blue) and DAPI (gray) and analyzed by confocal microscopy. (A,D-F) Injection of 13C-FITC (green). (A) Lymphoid follicle in the large intestine. Overlay of DAPI, 13C-FITC, and CD11C staining. (D-F) Detail of image shown in [(A); red square]. (B,C) Injection of the control peptide 13Csc-FITC (green). (B) Lymphoid follicle in the large intestine. Overlay of DAPI, 13Csc-FITC and CD11C staining. (C) Overlay of 13Csc-FITC and CD11c staining. (D) As (A); (E) CD11C staining; (F) 13C-FITC fluorescence. Pictures are representatives of three individual experiments with similar results. LP, lamina propria; L, lumen; gray, DAPI; blue, CD11C-Alexa647; green, FITC; scale in panels (A-C): $50 \mu \mathrm{m}$; scale in panels (D-F): $10 \mu \mathrm{m}$. 

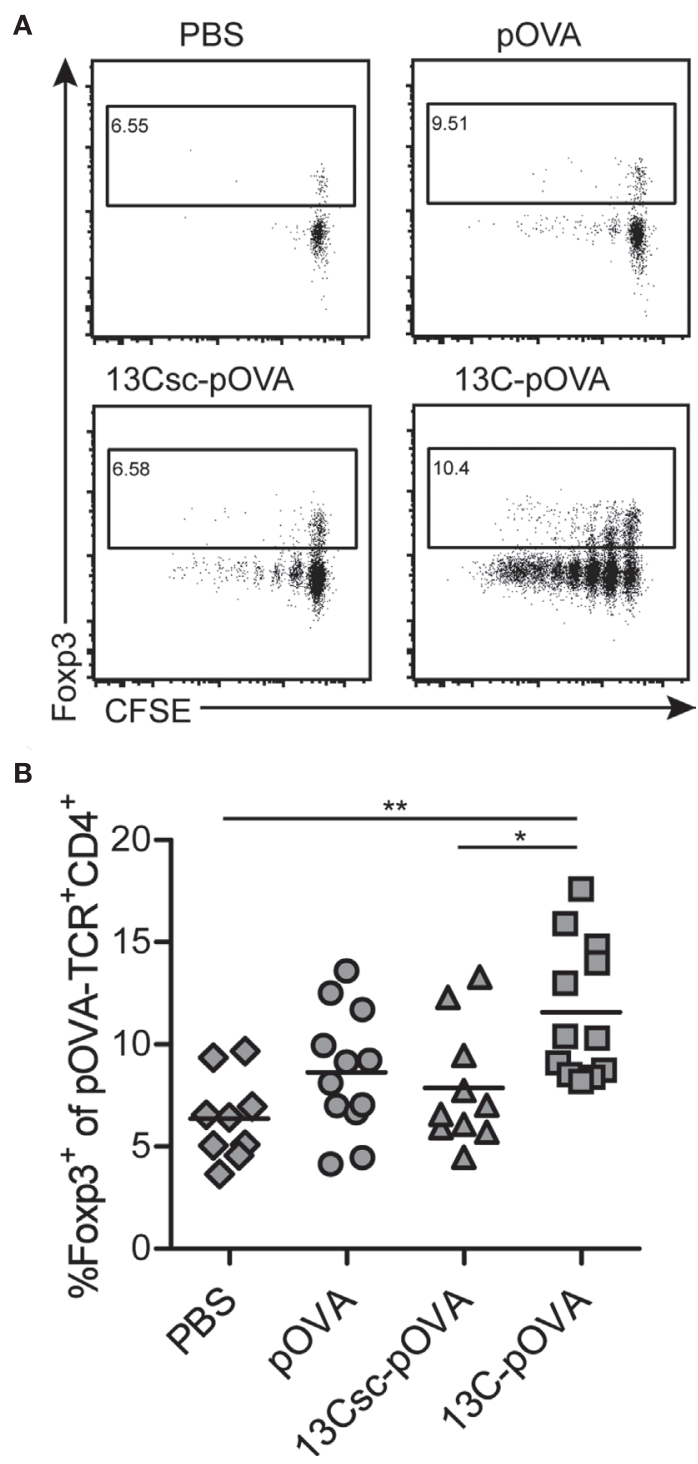

FIGURE 4 | 13C-pOVA treatment slightly increases the frequency of regulatory $\mathbf{T}$ cells after intrarectal treatment. Mice were treated as described in Figure $\mathbf{1}$ and isolated cells from mesenteric lymph nodes were analyzed by flow cytometry on day 3 after peptide treatment for the expression of Forkhead-box-protein 3 (Foxp3). (A) Exemplary FACS dot plots of CFSE dilution and expression of Foxp3 among pOVA-TCR ${ }^{+} \mathrm{CD} 4^{+} \mathrm{T}$ cells. (B) Frequency of antigen-specific Foxp $3^{+} \mathrm{T}$ cells. Pooled data from six individual experiments; symbols represent values from individual mice ( $n=9-12)$. Statistical testing was performed using One-way ANOVA $(p=0.0017)$ after confirming normality distribution using a D'Agostino and Pearson omnibus normality test ( $\alpha=0.05)$; statistical symbols represent results from Tukey's multiple comparison post test.

nasal application of $13 \mathrm{C}$ coupled to pOVA significantly increased in vivo proliferation of antigen-specific T cells compared to application of pOVA in at least some lymphoid tissues. This indicates that 13C-pOVA is taken up via the mucosal surfaces more efficiently than non-coupled pOVA, and functionally intact pOVA is delivered to and presented by APC. Consistent with the concept of shared properties of mucosal surfaces and a common mucosaassociated lymphoid tissue, immunization via the oral, nasal, and rectal route all were able to activate the pOVA-specific T cells in draining and systemic lymphoid tissues, albeit with quantitative and qualitative differences. Nasal peptide application proved to be most efficient in inducing $\mathrm{T}$ cell proliferation. A superiority of the nasal route for $\mathrm{T}$ cell priming has also been observed for protein treatment $(8,12)$. However, in the DTH model studied here, an equal or better tolerogenic efficacy of nasal application could not be confirmed.

Microscopic analysis revealed that the fluorochrome-labeled 13C transporter is taken up while the scrambled control peptide largely remains in the lumen of the large intestine, as already shown previously for the small intestine (4). Uptake is first observed in a subset of epithelial cells that have the morphological features of goblet cells, notably in the small intestine (4), but the conjugate also becomes detectable in mucosal CD $11 c^{+}$cells, which mainly consist of DCs (Figure 3). The proportion of the conjugates remaining in DCs and the proportion being transported via lymph to the systemic circulation could not been determined with the present technique. However, the proliferation of antigen-specific cells on day 3 after treatment was comparable in all analyzed lymphoid tissues and was not restricted to the draining LN. This indicates that a major part of the transported peptide is delivered intact into circulation and not only presented on mucosal APC. It can be assumed that the increased proliferation of $\mathrm{T}$ cells in mice treated with 13C-pOVA is a consequence of a synergistic effect of faster delivery of the peptide itself throughout the body and direct uptake of the peptides by APC at the site of application. Whether or not migration of peptide-loaded DCs from the mucosa into the draining lymph node contributes to the systemic dissemination of $\mathrm{T}$ cell-activating potential, and whether or not a more rapid initial response can be observed at draining sites remains to be determined.

To our surprise we noticed that also some native pOVA was able to cross the mucosal surfaces and elicit $\mathrm{T}$ cell responses in mucosal as well as remote lymphoid tissues such as spleen or SLN. Thus, a small fraction of antigenic peptides might naturally access the circulation in intact form; comparing the level of responses to peptide given by i.v. injection versus mucosal application, this fraction can be estimated to be less than $1 \%$. Nevertheless, coupling of pOVA to $13 \mathrm{C}$ peptide strongly improved the uptake.

It has been reported that feeding of ovalbumin protein in high doses leads to strong proliferation of the transferred antigenspecific T cells; however, the cells become anergic or undergo activation induced cell death (AICD) (13-15). In contrast, lowdose treatment with ovalbumin protein results in the induction of antigen-specific Tregs and, accordingly, this pathway has been seen as the predominant mechanism of low-dose tolerance (13, 14). However, this view has been challenged by other work, showing that prolonged high-dose treatment can also increase the number of Foxp $3^{+}$Tregs (16).

The treatment with $0.25 \mathrm{mg}(0.14 \mu \mathrm{mol})$ pOVA peptide used here is equivalent to approximately $6 \mathrm{mg}$ ovalbumin protein, i.e., a rather low-dose range. Accordingly, a significant increase in the frequency of Foxp $3^{+}$Tregs compared to the non-immunized 

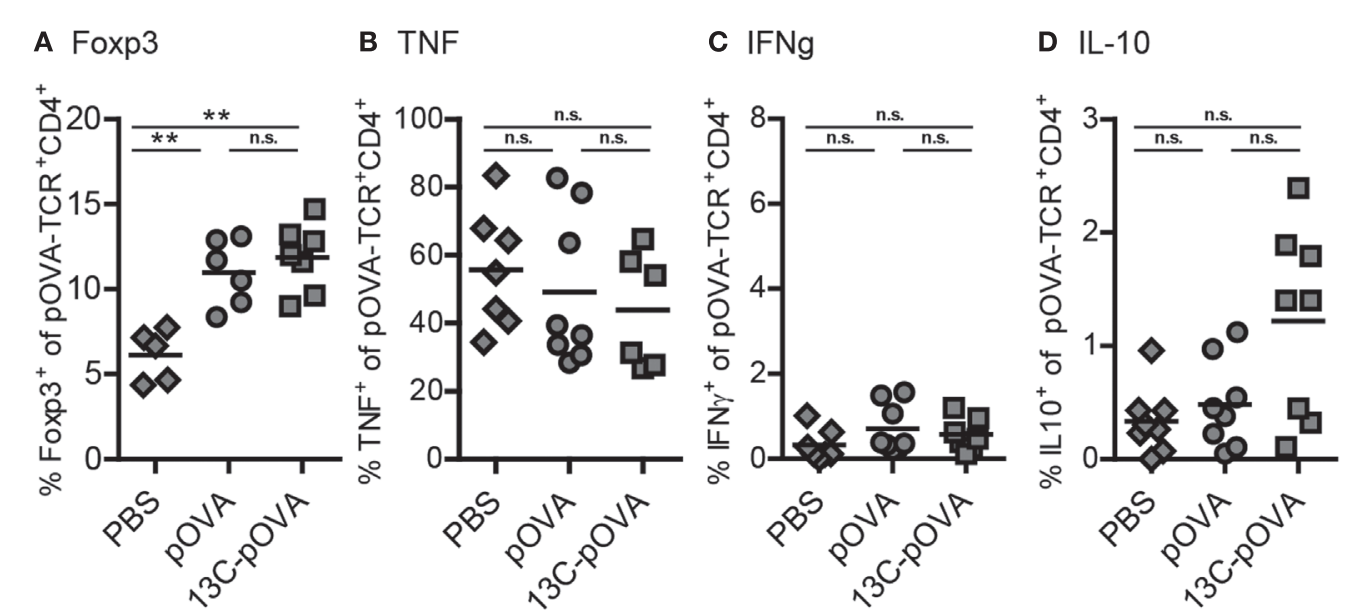

E IL-10/TNF

F

PBS
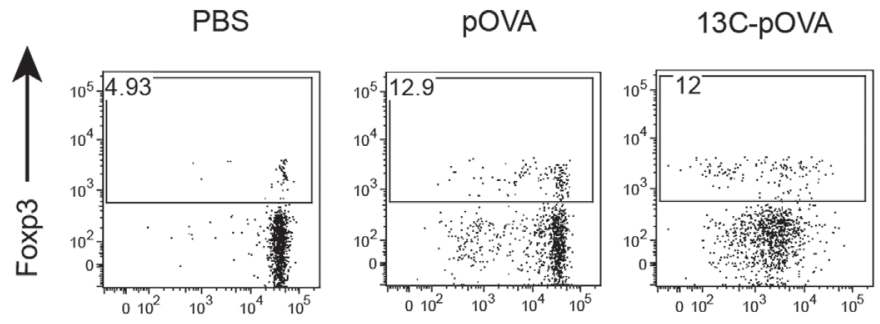

isotype control
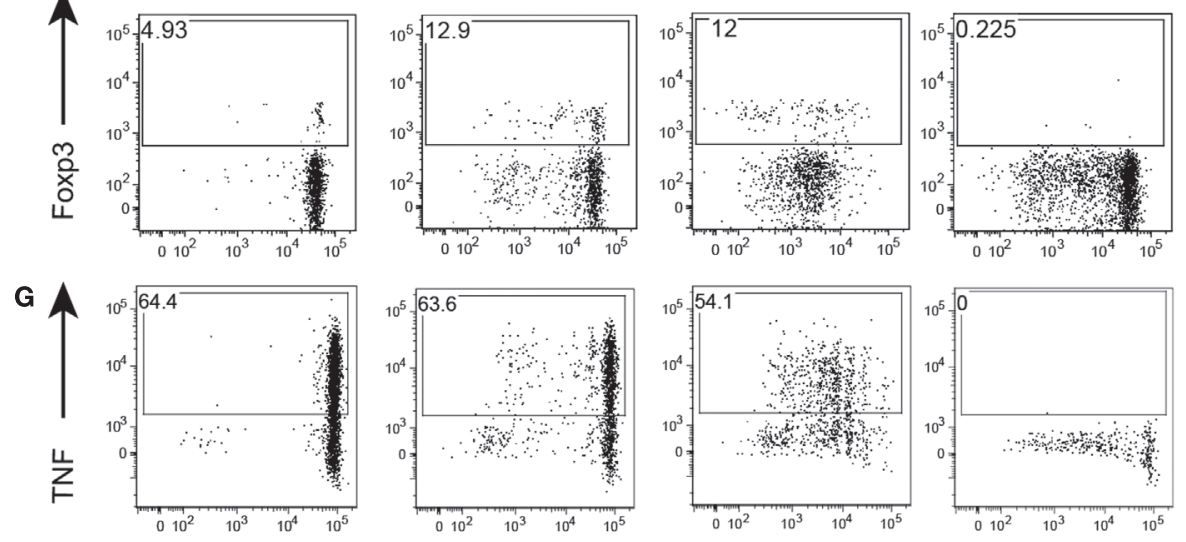

H
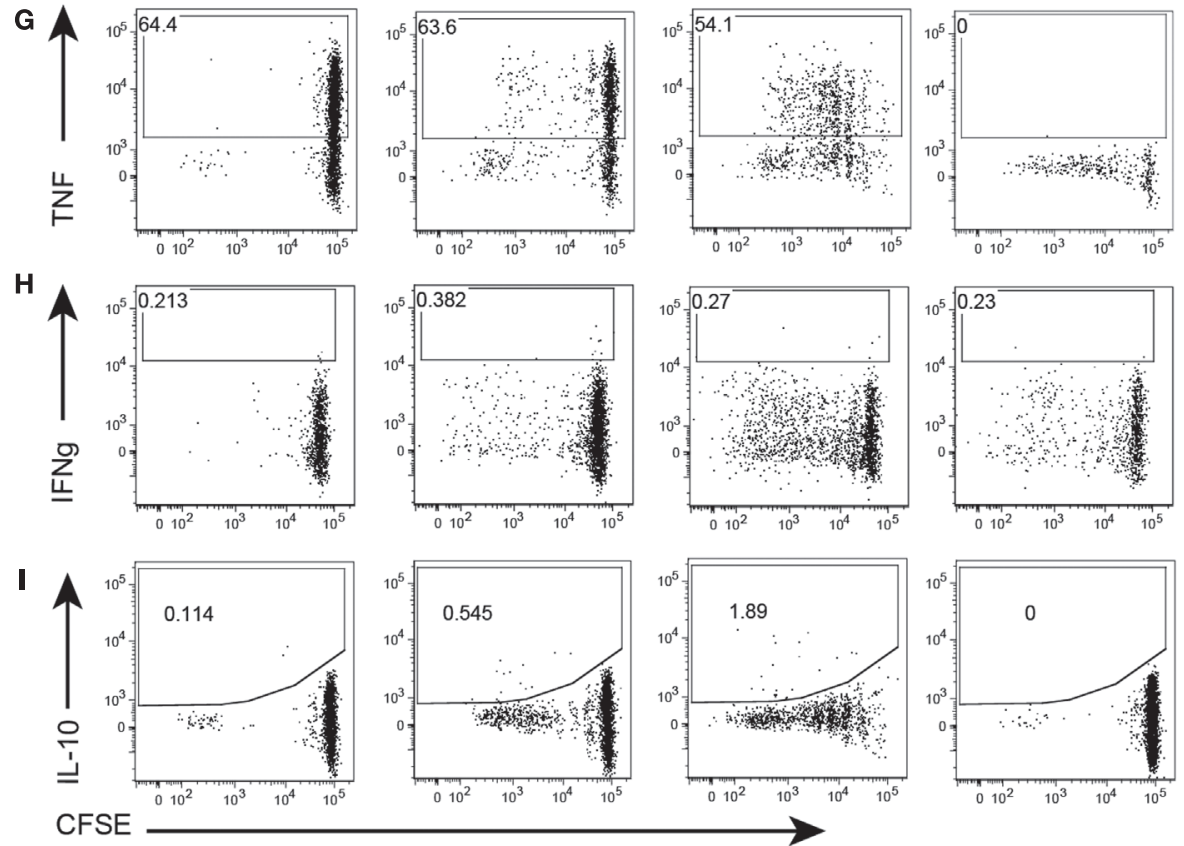

FIGURE 5 | Changes in production of pro- and anti-inflammatory cytokines. Mice were treated as described in Figure 1 and isolated cells from mesenteric lymph nodes were analyzed by flow cytometry on day 6 for the expression of Forkhead-box-protein 3 (Foxp3) and various intracellular cytokines. (A,F) Foxp3 frequency, (B,G) frequency of TNF producing, (C,H) frequency of IFN $\gamma$ producing, and $(\mathbf{D}, \mathbf{I})$ frequency of IL-10-producing pOVA-TCR ${ }^{+} \mathrm{CD} 4^{+} \mathrm{T}$ cells. (E) Ratio of T cells expressing IL-10 and TNF. (A-E) Pooled data from two to three individual experiments are shown; symbols represent values from individual mice $(n=5-8)$. Statistical testing was performed using the non-parametric Mann-Whitney test and Holm-Bonferroni correction for multiple comparisons. (F-I) Representative dot plots.

animals was recorded, albeit the effect was not as pronounced as in other studies of oral immunization (16). However, despite strong $\mathrm{T}$ cell proliferation, indicated by loss of CFSE staining, the frequencies of transgenic T cells had only slightly increased at day 3 (Figure S3 in Supplementary Material). Thus, a significant part of the proliferating cells appears to undergo AICD. While only 


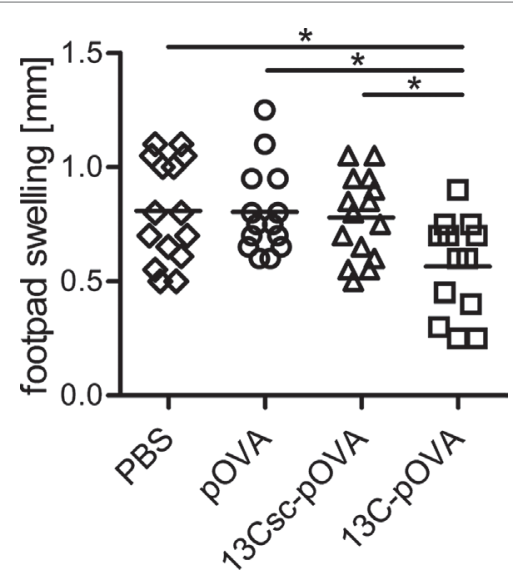

FIGURE 6 | Reduced footpad swelling in delayed-type hypersensitivity after tolerization with 13C-pOVA peptide. Balb/c mice were injected with $5 \times 10^{6}$ CFSE-labeled CD4 ${ }^{+}$cells from D011.10 donor mice. Twenty-four hours later, the mice were tolerized intrarectally with $0.25 \mathrm{mg}(0.14 \mu \mathrm{mol})$ of pOVA or equimolar amounts of 13C-pOVA or 13Csc-pOVA or phosphatebuffered saline (PBS) as control. On day 8, the mice were immunized by tail-base injection of pOVAVCFA emulsion. On day 22, the mice were injected with pOVA/IFA emulsion into the right footpad (the left footpad received PBS/ IFA as control). The following day, footpad swelling was measured. The graph shows the footpad swelling of the IFA/pOVA injected foot compared to the control foot. Pooled data from three individual experiments are shown; symbols represent values from individual mice $(n=13-15)$. Statistical testing was performed using One-way ANOVA ( $p=0.0082)$ after confirming normality distribution via D'Agostino and Pearson omnibus test $(\alpha=0.05)$; statistical symbols in graph represent result from Tukey's multiple comparisons post test.

a minor, non-significant reduction in TNF-producing effector cells was observed upon immunization with peptides, 13C-pOVA induced an increase in IL-10 producing $\mathrm{T}$ cells and hence in the ratio IL-10/TNF, suggesting that the peptide immunization via the mucosal route increased IL-10-mediated regulatory pathways.

Both IL-10 producing T cells (“TR1") and Foxp $3^{+}$, Tregs have been implicated in the suppression of inflammation in DTH or other models $(17,18)$. Indeed, in a DTH model, mucosal application of 13C-pOVA, but not pOVA, resulted in a significant, yet only partial suppression of footpad swelling upon rectal vaccination, and a trend to reduced inflammation upon oral and nasal vaccination (Figure S9 in Supplementary Material). Mucosal vaccination with 13C-pOVA in this model resulted consistently in increased frequencies of Tregs, while the effects on cytokines were less clear after immunization and challenge. A definite analysis of the pathways causing the partial suppression of the DTH requires blocking/deletion experiments, which was beyond the scope of the present study. In previous studies, tolerization in the DTH model was achieved by feeding high doses of ovalbumin protein (18). At the peptide dose applied here, only pOVA coupled to $13 \mathrm{C}$

\section{REFERENCES}

1. Faria AM, Weiner HL. Oral tolerance. Immunol Rev (2005) 206:232-59. doi:10.1111/j.0105-2896.2005.00280.x was able to significantly modulate the DTH response. Using this model of a strongly inflammatory disease, it can be concluded that the enhanced uptake of the transporter-coupled antigenic peptide led to more vigorous $\mathrm{T}$ cell proliferation, without increased effector cell frequencies, and some increase in antigen-specific Tregs. However, it has to be emphasized that the delivery of antigenic peptide by the mucosal route by itself is not as effective in inducing a robust tolerance as might have been expected. Whether this can be improved by altering conditions (e.g., repeated vaccination) and whether a clear preference for one of the mucosal application routes can be secured, remains to be shown.

We have previously demonstrated that the $13 \mathrm{C}$ peptide is also able to carry large proteins in intact form through the mucosal barrier (4). It will be interesting to test whether tolerization with intact proteins using this targeting pathway leads to different results compared with high-dose oral protein application, where most likely only peptides reach the circulation. The ability to deliver macromolecules via the mucosal barrier by virtue of the 13C peptide also provides novel opportunities of combining antigens with either tolerogenic or immunogenic adjuvant components, e.g., by coupling additionally either immunosuppressive or stimulatory cytokines to the antigen-transporter complex. Thus, the carrier peptide $13 \mathrm{C}$ might become a valuable component in a toolbox of functional units to be used for engineering novel immunomodulatory agents.

\section{AUTHOR CONTRIBUTIONS}

$\mathrm{EK}, \mathrm{JP}, \mathrm{UH}$, and $\mathrm{AH}$ conceived and designed the experiments. EK, JP, AK, and UL performed the experiments. EK, JP, and AK analyzed the data. EK, AP, and AH wrote and edited the manuscript.

\section{ACKNOWLEDGMENTS}

The authors would like to thank Prof. Dr. Rudolf Volkmer and his group at the Charité Universitätsmedizin Berlin for the support in peptide synthesis and the lab managers in the DRFZ for technical support. The authors would also like to thank Simone Spieckermann for expert technical assistance.

\section{FUNDING}

This work was supported by the Deutsche Forschungsgemeinschaft (SFB633 and SFB650) and partly funded by AgResearch Ltd. EK was supported by the ZIBI graduate school.

\section{SUPPLEMENTARY MATERIAL}

The Supplementary Material for this article can be found online at http://journal.frontiersin.org/article/10.3389/fimmu.2017.00216/ full\#supplementary-material.

2. Garside P, Mowat AM. Oral tolerance. Semin Immunol (2001) 13(3):177-85. doi:10.1006/smim.2001.0310

3. Issa F, Wood KJ. Translating tolerogenic therapies to the clinic - where do we stand? Front Immunol (2012) 3:254. doi:10.3389/fimmu.2012.00254 
4. Kenngott EE, Cole S, Hein WR, Hoffmann U, Lauer U, Maass D, et al. Identification of targeting peptides for mucosal delivery in sheep and mice. Mol Pharm (2016) 13(1):202-10. doi:10.1021/acs.molpharmaceut. 5 b00635

5. McDole JR, Wheeler LW, McDonald KG, Wang B, Konjufca V, Knoop KA, et al. Goblet cells deliver luminal antigen to CD103+ dendritic cells in the small intestine. Nature (2012) 483(7389):345-9. doi:10.1038/nature10863

6. Knoop KA, McDonald KG, McCrate S, McDole JR, Newberry RD. Microbial sensing by goblet cells controls immune surveillance of luminal antigens in the colon. Mucosal Immunol (2015) 8(1):198-210. doi:10.1038/mi.2014.58

7. Kearney ER, Pape KA, Loh DY, Jenkins MK. Visualization of peptide-specific $\mathrm{T}$ cell immunity and peripheral tolerance induction in vivo. Immunity (1994) 1(4):327-39. doi:10.1016/1074-7613(94)90084-1

8. Faria AM, Weiner HL. Oral tolerance: therapeutic implications for autoimmune diseases. Clin Dev Immunol (2006) 13(2-4):143-57. doi:10.1080/ 17402520600876804

9. Coombes JL, Siddiqui KR, Arancibia-Carcamo CV, Hall J, Sun CM, Belkaid $\mathrm{Y}$, et al. A functionally specialized population of mucosal CD103+ DCs induces Foxp3+ regulatory $\mathrm{T}$ cells via a TGF-beta and retinoic acid-dependent mechanism. J Exp Med (2007) 204(8):1757-64. doi:10.1084/jem. 20070590

10. Mucida D, Kutchukhidze N, Erazo A, Russo M, Lafaille JJ, Curotto de Lafaille MA. Oral tolerance in the absence of naturally occurring Tregs. J Clin Invest (2005) 115(7):1923-33. doi:10.1172/JCI24487

11. Sun CM, Hall JA, Blank RB, Bouladoux N, Oukka M, Mora JR, et al. Small intestine lamina propria dendritic cells promote de novo generation of Foxp3 T reg cells via retinoic acid. J Exp Med (2007) 204(8):1775-85. doi:10.1084/ jem.20070602

12. Hanninen A, Braakhuis A, Heath WR, Harrison LC. Mucosal antigen primes diabetogenic cytotoxic T-lymphocytes regardless of dose or delivery route. Diabetes (2001) 50(4):771-5. doi:10.2337/diabetes.50.4.771

13. Weiner HL, da Cunha AP, Quintana F, Wu H. Oral tolerance. Immunol Rev (2011) 241(1):241-59. doi:10.1111/j.1600-065X.2011.01017.x
14. Chen Y, Inobe J, Kuchroo VK, Baron JL, Janeway CA Jr, Weiner HL. Oral tolerance in myelin basic protein T-cell receptor transgenic mice: suppression of autoimmune encephalomyelitis and dose-dependent induction of regulatory cells. Proc Natl Acad Sci U S A (1996) 93(1):388-91. doi:10.1073/ pnas.93.1.388

15. Chen Y, Inobe J, Marks R, Gonnella P, Kuchroo VK, Weiner HL. Peripheral deletion of antigen-reactive $\mathrm{T}$ cells in oral tolerance. Nature (1995) 376(6536):177-80. doi:10.1038/376177a0

16. Siewert C, Lauer U, Cording S, Bopp T, Schmitt E, Hamann A, et al. Experience-driven development: effector/memory-like alphaE+Foxp3+ regulatory $\mathrm{T}$ cells originate from both naive $\mathrm{T}$ cells and naturally occurring naive-like regulatory T cells. J Immunol (2008) 180(1):146-55. doi:10.4049/ jimmunol.180.1.146

17. Li L, Elliott JF, Mosmann TR. IL-10 inhibits cytokine production, vascular leakage, and swelling during $\mathrm{T}$ helper 1 cell-induced delayed-type hypersensitivity. J Immunol (1994) 153(9):3967-78.

18. Blumenthal-Barby F, Schrage A, Eulenburg K, Zeitz M, Hamann A, Klugewitz K. Sustained delayed-type hypersensitivity reaction after in vivo priming but successful induction of unresponsiveness after adoptive transfer of CD4+ effector T cells. Cell Immunol (2008) 253(1-2):110-5. doi:10.1016/j. cellimm.2008.05.004

Conflict of Interest Statement: The authors declare that the research was conducted in the absence of any commercial or financial relationships that could be construed as a potential conflict of interest.

Copyright (c) 2017 Kenngott, Pfeil, Hoffmann, Lauer, Kühl, Rigby, Pernthaner and Hamann. This is an open-access article distributed under the terms of the Creative Commons Attribution License (CC BY). The use, distribution or reproduction in other forums is permitted, provided the original author(s) or licensor are credited and that the original publication in this journal is cited, in accordance with accepted academic practice. No use, distribution or reproduction is permitted which does not comply with these terms. 